\title{
Identification of Accretion as Grain Growth Mechanism in Astrophysically Relevant Water-Ice Dusty Plasma Experiment
}

\author{
Ryan S. Marshall, Kil-Byoung Chai ${ }^{1}$, and Paul M. Bellan \\ Applied Physics and Materials Science, Caltech, Pasadena, CA 91125, USA \\ Received 2016 June 15; revised 2017 January 24; accepted 2017 January 26; published 2017 March 2
}

\begin{abstract}
The grain growth process in the Caltech water-ice dusty plasma experiment has been studied using a high-speed camera and a long-distance microscope lens. It is observed that (i) the ice grain number density decreases fourfold as the average grain major axis increases from 20 to $80 \mu \mathrm{m}$, (ii) the major axis length has a log-normal distribution rather than a power-law dependence, and (iii) no collisions between ice grains are apparent. The grains have a large negative charge resulting in strong mutual repulsion and this, combined with the fractal character of the ice grains, prevents them from agglomerating. In order for the grain kinetic energy to be sufficiently small to prevent collisions between ice grains, the volumetric packing factor (i.e., ratio of the actual volume to the volume of a circumscribing ellipsoid) of the ice grains must be less than $\sim 0.1$ depending on the exact relative velocity of the grains in question. Thus, it is concluded that direct accretion of water molecules is very likely to dominate the observed ice grain growth.
\end{abstract}

Key words: dust, extinction - methods: laboratory: solid state - planets and satellites: rings - plasmas protoplanetary disks

Supporting material: animations

\section{Introduction}

Very weakly ionized plasma with water-ice grains occurs in many parts of the universe. On Earth these water-ice dusty plasmas are a key component of polar mesospheric clouds (Havnes et al. 1996). Saturn's diffuse E, F, and G rings are a much larger water-ice dusty plasma (Goertz 1989). On an even larger scale, protoplanetary disks and molecular clouds are comprised of very weakly ionized plasma with dusts including water-ice grains (Sano et al. 2000).

The ice grain growth process is difficult to observe directly in protoplanetary disks and molecular clouds since these structures are distant and presumably have a growth time much longer than the human scale. Therefore, indirect, survey-type telescope measurements of multi-wavelength emissions ranging from micrometer to millimeter are typically used to estimate the growth process of dusts including ice grains (Andrews \& Williams 2005; Ricci et al. 2010; Ubach et al. 2012). An observed spectral energy distribution is fitted in these estimates to a standard dust emission model to obtain the maximum or mean size of dusts; these estimates assume that dust grains are spherical and the dust density has a powerlaw dependence on the radius. The ice dust grain growth is then deduced by sorting the observed data as a function of the disk age or the evolution stage. Based on the constraints from multiwavelength observations and laboratory experiments (with non-ice dusts and non-plasma environments) (Blum et al. 2000; Poppe et al. 2000; Schrapler \& Blum 2011) several models have been developed to explain planetesimal formation (Brauer et al. 2008; Birnstiel et al. 2012; Okuzumi et al. 2012). The process that has been proposed is that ice grains are heterogeneously nucleated on refractory materials (Greenberg 1982) and that they quickly grow to millimeter or

\footnotetext{
${ }^{1}$ Current Address: Korea Atomic Energy Research Institute, 989-111 Daedeok-daero, Yuseong-gu, Daejeon, Korea.
}

centimeter size in the outer disk regions (Andrews \& Williams 2005; Ricci et al. 2010; Testi et al. 2014).

In contrast to ice grains observed in astrophysical contexts, water-ice grains in polar mesospheric clouds are presumed to nucleate on meteorite smoke particles and grow by accreting water molecules (Turco et al. 1982), but the actual growth process has not been observed. Rocket-borne detectors measured the water-ice grain size to be a few tens of nanometers and the charge to be about one electron (Havnes et al. 1996).

Ice grains in Saturn's E-ring are presumed to grow because water molecules and small water-ice grains are continuously ejected from the South Pole of Enceladus (Porco et al. 2006). There is no obvious local water source other than the E-ring for ice growth to occur. The Cassini spacecraft's Plasma Spectrometer and Cosmic Dust Analyzer measures the size, speed, and charges of ice grains in Saturn's E-ring and Enceladus plume. These measurements indicate that the size of ice grains is $<10 \mu \mathrm{m}$ and that most ice grains are negatively charged, while a few are positively charged (Kempf et al. 2008; Jones et al. 2009; Hill et al. 2012). Direct observation of ice grain growth is not currently available.

Dust charging has typically been ignored in astrophysical situations such as protoplanetary disks and molecular clouds, presumably because the plasma density is assumed to be low compared to the dust density. However, the ratio of the plasma to dust density might not be small in the outer disk regions (Sano et al. 2000; Okuzumi 2009; Matthews et al. 2012) where ice grains are postulated to grow quickly to millimeter size and the ice grains are likely to be charged. Charging occurs because the ice grains are continuously bombarded by electrons and ions that collide with and attach to the ice grains. If all ice grains are charged with the same electric polarity, there would be an electrostatic repulsive force between the grains and this force would oppose agglomeration of the ice grains. It is very important to note that although this electrostatic repulsive force between ice grains impedes agglomeration, it does not impede 


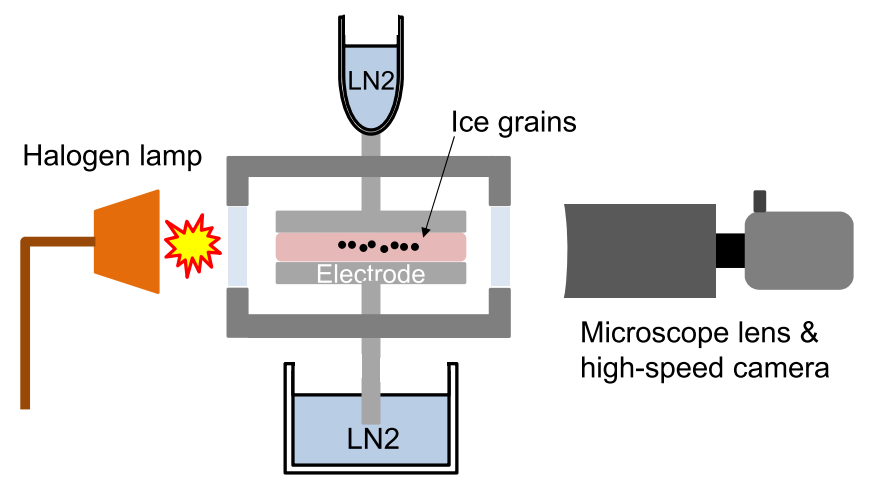

Figure 1. A sketch of experimental setup.

another important means of ice grain growth. In particular, if the ice grains grow via accretion of neutral water molecules, a mechanism that we call accretion growth, then the growth rate of the ice grains will increase as the grains become more charged. This is because neutral water molecules have a very large dipole moment and so are attracted to charged ice grains in proportion to the degree of grain charging. Thus, increasing the ice grain charge will accelerate growth by accretion and decelerate growth by agglomeration.

The role of electrical charge in ice dust grain growth can be discerned by examining the dust growth process in laboratory dusty plasmas ignited with reactive gases such as $\mathrm{SiH}_{4}$ (Boufendi \& Bouchoule 1994; Dorier et al. 1995), $\mathrm{C}_{2} \mathrm{H}_{2}$, and $\mathrm{CH}_{4}$ (Hong et al. 2003). It is generally accepted that nanometer sized dust particles in these plasmas form spontaneously and then proceed to grow by continually colliding with each other (Boufendi \& Bouchoule 1994; Hong et al. 2003). This growth by coagulation ceases when the ice grains become micron sized and so have acquired enough electric charge for mutual repulsion to prevent further coagulation. After this growth by coagulation, dust particles can grow further by accreting molecules and ions (Boufendi \& Bouchoule 1994; Dorier et al. 1995; Hong et al. 2003). However, it has been recently reported that dust grains larger than micrometers immersed in a plasma can grow by the agglomeration process, even though they have large electric charges (Du et al. 2010; Yousefi et al. 2014). In order for this agglomeration growth to occur in laboratory plasmas, the kinetic energy of a dust particle must overcome the Coulomb repulsive potential energy; dust acoustic waves have been proposed as a mechanism for providing the required kinetic energy (Du et al. 2010; Yousefi et al. 2014).

The Caltech water-ice dusty plasma experiment provides a unique opportunity to observe directly how ice grains in a plasma environment grow from a few micrometers to hundreds of micrometers. A long-distance microscope lens mounted on a fast movie camera shows this growth in detail. Similar to the terrestrial and extraterrestrial applications, the Caltech dusty plasma is weakly ionized $\left(\sim 10^{-6}\right.$ ionization fraction) and the dust consists of water-ice grains. The temperature is below 150 $\mathrm{K}$, which is similar to the temperature of naturally occurring ice dusty plasmas. The ice grains are observed to be elongated and fractal. We will provide observational evidence that electrical charge is sufficiently large to prevent growth by agglomeration so that growth by accretion dominates.

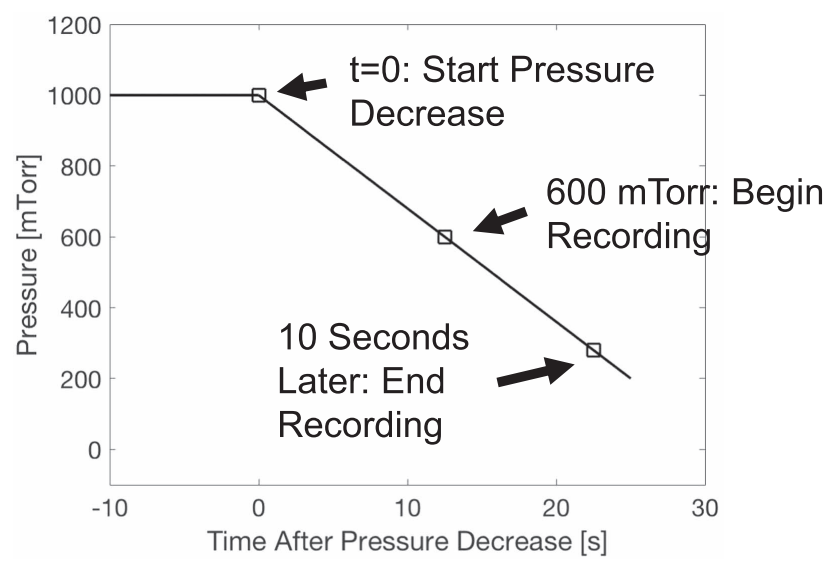

Figure 2. Plot of pressure in vacuum chamber as a function of time after pressure decrease.

\section{Caltech Ice Dusty Plasma Experimental Setup}

Figure 1 shows the water-ice dusty plasma apparatus; more detail is given in Chai \& Bellan (2013). An important difference from Chai \& Bellan (2013) is that the electrodes have been changed to be copper instead of aluminum because copper is more thermally conductive. This change results in colder electrodes because the liquid nitrogen cooling is more effective.

A $500 \mathrm{~W}$ halogen lamp shines through one window of the vacuum chamber to back-illuminate water-ice grains formed in helium plasma. High-speed video of a cluster of individual ice grains is recorded using an Infinity K2 DistaMax long-distance microscope lens attached to a Photron SA-X2 high-speed camera viewing the chamber interior through an oppositely facing window. This setup provides an approximately $200 \mu \mathrm{m}$ depth of field and $1.5 \times 1.5 \mathrm{~mm}^{2}$ field of view. The light scattered from the ice grains makes the grains appear as dark particles on a light background.

The experimental procedure is similar to Chai \& Bellan (2013). After purging residual gas from the distilled water tank, liquid nitrogen is poured into the upper and lower reservoirs and the electrodes are allowed to cool for 30 minutes. The main vacuum chamber is then filled with 1 Torr helium gas and the plasma is ignited by application of $1-3 \mathrm{~W}$ of $13.56 \mathrm{MHz}$ RF power across the electrodes. After plasma ignition, water vapor is introduced into the plasma. The amount of water vapor is gauged by the increase in reading of an MKS Capacitance Manometer. The increase in the manometer reading is 20 mTorr, which implies the ratio of the helium partial pressure to the water vapor partial pressure is 50 assuming that this increase is solely from water vapor and not from air. Immediately upon introduction of the water vapor, water-ice grains spontaneously form and grow. Ice grain formation is only observed when the ambient helium pressure exceeds 600 mTorr. After growth begins, the ambient gas pressure is lowered from 1 Torr to 200 mTorr as illustrated in Figure 2. When the ambient helium pressure has decreased to 600 mTorr, the ice grains have become macroscopic and the video recording of the ice grain growth process is started. The camera records a $10 \mathrm{~s}$ long video at 4000 frames per second. During the recording time, the pressure drops to 200 mTorr while the ice grains grow from 20 to $80 \mu \mathrm{m}$. The 40,000 recorded photos were analyzed and the results will now be presented and discussed. 


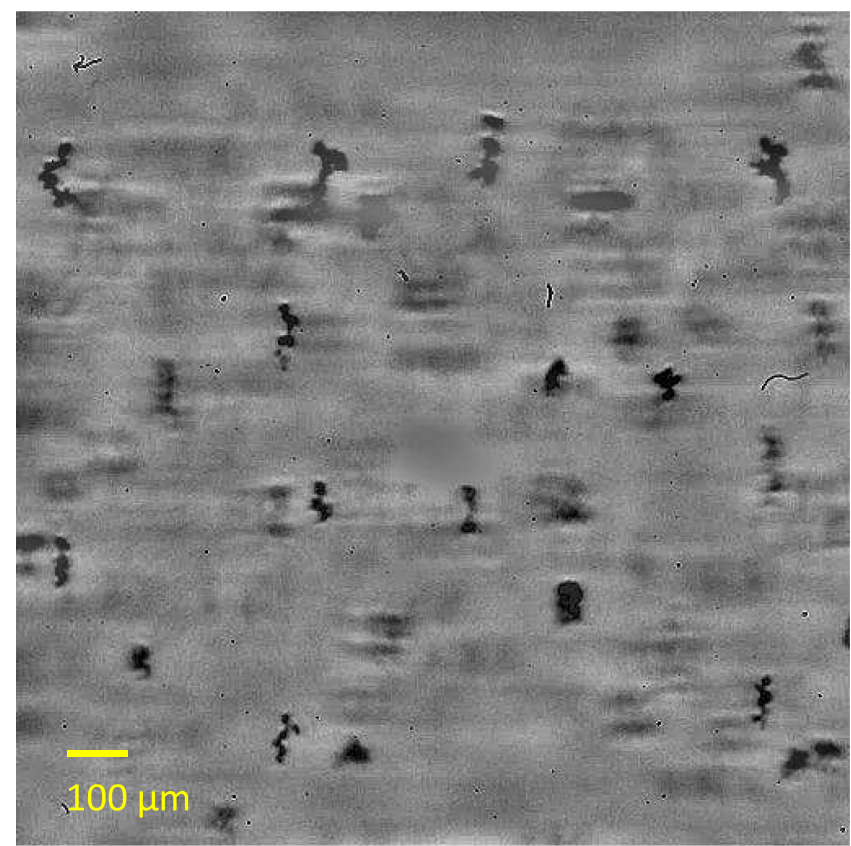

Figure 3. Still image frame taken from the high-speed movie. A sample of the high-speed movie, consisting of 6 segments of 400 frames with 2 s intersegment time, can be seen in the online version at $10 \mathrm{fps}$. The frames in the original movie are 1.45 megabytes each, so the 40,000 frames are 58.4 gigabytes. Because this file size greatly exceeds the 100 megabyte upload limit, the frames in the online sample movie have been compressed to 29 kilobytes each, so that the 2400 frame movie is 70 megabytes. This compromise of compression and segmentation provides both reasonable resolution and reasonable representation of the growth. The original high-resolution movie was used for the actual data analysis.

(An animation of this figure is available.)

\section{Analysis of High-speed Video}

Watching the 4000 frames per second video at 10 frames per second provides insights into the ice grain motion and growth process that would not otherwise be evident without the 400fold slowdown of the motion. Figure 3 (the movie version can be found in the online journal) shows a sample of the highspeed video. The exact trajectory of each ice grain can be identified and followed for over 50 frames and sometimes as many as 250 or more frames. The $10 \mathrm{~s}$ long video shows a considerable growth in particle size. This is in agreement with Chai \& Bellan (2013) where it was reported that when ambient pressure decreases, ice grain size increases. As the ice grains grow, a decrease in the overall ice grain number density in the plasma is also observed.

The 4000 per second frame rate is sufficient to follow oscillatory ice grain motion possibly caused by dust acoustic waves. Because the position of each ice grain is measured as a function of time, the velocity of each ice grain can be computed. This computation was done for every ice grain between all consecutive pairs of frames to create an evolving temporal speed distribution. It was furthermore possible to observe ice grains, vertically aligned as in Chai \& Bellan (2015a), spinning about their vertical axis. In rarer instances, an ice grain would be observed tumbling and spinning about some other axis or combination of axes.

A critical result is that no direct collisions between ice grains were observed in the 40,000 frame video inside the camera's field of view. It is important to note that this does not preclude the possibility that collisions could occur outside the field of view. Moreover, it was not even possible to locate an obvious example where two ice grains approached on a collision course and then deflected from each other according to Rutherford scattering. There is no observational evidence of two ice grains colliding and sticking to each other, nor is there any evidence of two ice grains colliding and breaking apart. This lack of evidence for collisions between grains suggests that accretion is the dominant mode of growth, not coagulation.

\subsection{Ice Grain Size, Aspect Ratio, and Number Density}

The solid line in Figure 4(a) shows the time evolution of the major axis length. This measurement was determined by a three-step process: every 10th video image was read into a Matlab code and sharpened to make the ice grains more distinct. The images were then filtered according to particle size and partial ice grains cut off at the edges were removed. The built-in Matlab function regionprops was then used to calculate the centroid, major axis, and minor axis lengths of all the remaining ice grains in each image. The major axis values from each ice grain in the frame were then averaged and plotted. Figure 4(a) demonstrates that the water-ice grains grew from 20 to $80 \mu \mathrm{m}$ at an almost constant rate as the ambient pressure decreased from 600 mTorr to 200 mTorr during $10 \mathrm{~s}$.

Since regionprops does not give an accurate minor axis value, a different method was used to investigate the major and minor axis lengths as a function of time. This was accomplished by selecting six frames from the video corresponding to different times and physically measuring the length in pixels of the major and minor axes of a number of ice grains in each using the software ImageJ. Only the particles that were in sufficiently sharp focus to obtain accurate dimensions were used; this selection resulted in a sample size of between 16 and 32 ice grains for each frame. These hand-measured major axis lengths, indicated by square dots in Figure 4(a), show the same trend and very similar values to the regionprops data as the grain length increased from 40 to $80 \mu \mathrm{m}$. Meanwhile, the minor axis length indicated by circular dots in Figure 4(a) increased from 10 to $20 \mu \mathrm{m}$ between 4 and $10 \mathrm{~s}$.

The average aspect ratio for each frame was calculated by dividing each respective major and minor axis length and is shown in Figure 4(b). It is apparent that the aspect ratio stayed roughly constant at $\sim 4.25$ throughout the growth process.

The number of ice grains in a frame determined by regionprops as a function of time is shown as the solid line in Figure 5. In order to determine whether or not the data from regionprops are accurate, the number of ice grains in a frame is physically counted from 10 selected frames and is displayed as the circles in Figure 5. The hand-measured numbers show the same trend and similar values to the regionprops data as the number of grains decreases from 120 to 30 . These data can be used to quantify the water-ice grain number density because the number of ice grains in each frame is proportional to the number density in the plasma. The constant of proportionality is determined from the size of the frame $\left(1.5 \times 1.5 \mathrm{~mm}^{2}\right)$ and the depth of field of the regionprops function $(1 \mathrm{~mm})$; note that the regionprops function has a thicker depth of field than the camera system because regionprops logs out of focus particles outside the depth of view of the lens. The number density of ice grains decreased roughly linearly with time over most of the growth process.

The number density decreased by a factor of four, while the ice grain major radius increased by a factor of four. Section 4.1 

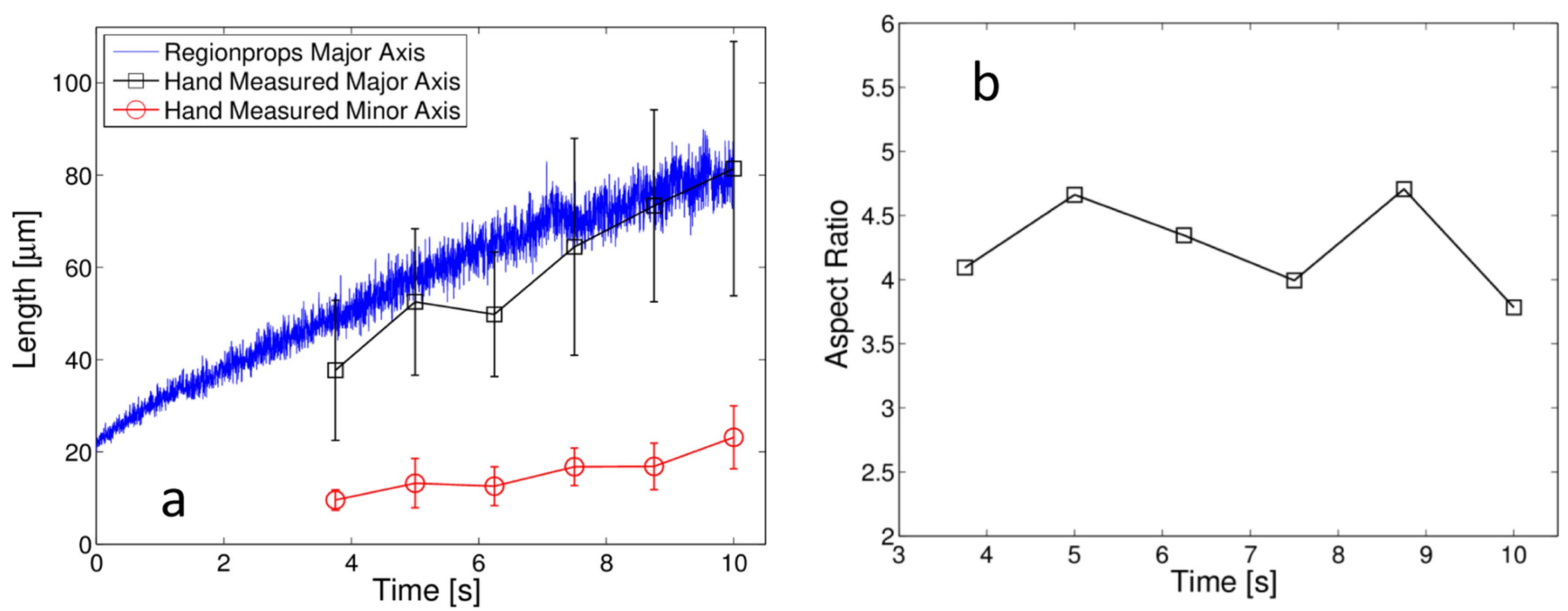

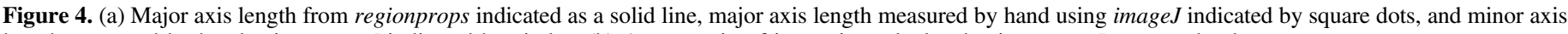
length measured by hand using imageJ indicated by circles. (b) Aspect ratio of ice grains calculated using imageJ measured values.

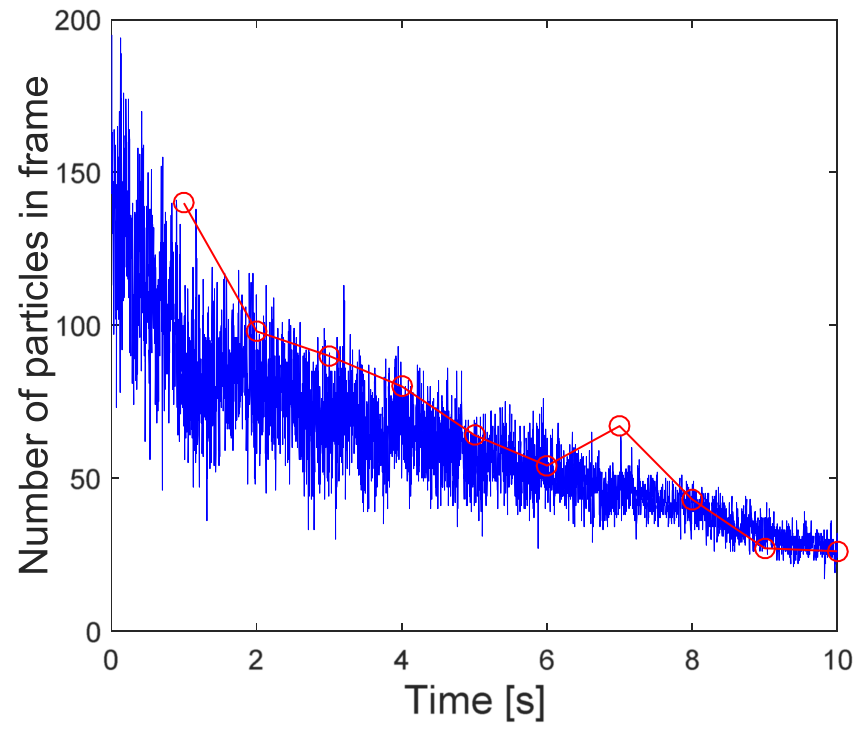

Figure 5. Time evolution of the number of particles in each frame.

discusses the significance of the factor of four number density decrease being small compared to what might be expected from the observed change in total ice grain volume, which increases by a factor of $r^{d}$ where $d$ is the fractal dimension of the ice grains. Section 4.1 explains why this small decrease in number density supports the conclusion that growth by accretion dominates in this experiment.

\subsection{Major Axis Length Distribution}

The major axis length of all ice grains in each frame obtained from the regionprops function can be plotted as a histogram. Figures 6(a)-(d) show the major axis length histogram at 2.5, 5, 7.5 , and $10 \mathrm{~s}$. The $x$-axis is the major axis length in $\mu \mathrm{m}$ and the $y$-axis is the number of particles. As seen in Figure 6, both the mean value of the major length and the FWHM of the distribution function increase with time. The distributions are approximately log-normal which significantly differs from the power-law distribution typically assumed in astrophysical contexts (Andrews \& Williams 2005; Brauer et al. 2008; Ricci et al. 2010; Birnstiel et al. 2012). This difference in distribution is presumed to result from the growth primarily coming from accretion as opposed to agglomeration. We note that the powerlaw distribution assumed in previous astrophysical contexts is based on the assumption that an equilibrium develops between the collisional processes of agglomeration and fragmentation. If the dust grains do not collide, there is no mechanism for establishing the power-law distribution.

\subsection{Oscillation Motion and Velocity Distribution}

The 4000 frames per second video from the Photron SA-X2 camera enabled the tracking of trajectories of individual ice grains. At each frame, the $(x, y)$ position of any specific ice grain was recorded. Figure 7 (a) shows the $(x, y)$ trajectory of a particle starting from frame 20,000 corresponding to time $5 \mathrm{~s}$ while Figure 7(c) shows another trajectory starting from frame 35,000 corresponding to time $8.75 \mathrm{~s}$. In each case, the particle in question was followed for 100 frames and no collisions were observed. Using these position coordinates and the $0.25 \mathrm{~ms}$ interframe time, the speed of the ice grain was calculated. The ice grain speed for the trajectory starting at $5 \mathrm{~s}$ is shown in Figure 7(b) and the speed of the ice grain for the trajectory starting at $8.75 \mathrm{~s}$ is shown in Figure $7(\mathrm{~d})$. The speed is found using $v=\left(\sqrt{(\Delta x)^{2}+(\Delta y)^{2}} \times 2.7 \mu \mathrm{m}\right) /(0.25 \mathrm{~ms})$ where $\Delta x$ is the difference in pixels of the $x$ position of the ice grain between two consecutive frames and $\Delta y$ is the same for the $y$ position; $2.7 \mu \mathrm{m}$ is the distance between pixels.

Figure 7(e) plots the vertical oscillation frequency of the ice grains as a function of time through the growth process. The frequency was found by watching a pair of well-defined ice grains oscillate through one whole period. The overall trend is that as the ice grains grow, the oscillation frequency drops from 260 to $130 \mathrm{~Hz}$.

It is possible that the observed periodic motion of ice grains in the vertical direction is a result of dust acoustic waves and the horizontal dark regions in the movie are wave fronts. However, because the camera system field of view is too small to observe more than two of these wave fronts, it is not possible to conclude for certain that the dark regions are dust acoustic waves.

The speed distributions of ice grains at 2.5, 5, 7.5, and $10 \mathrm{~s}$ are shown in Figures 8(a)-(d), respectively; these distributions 

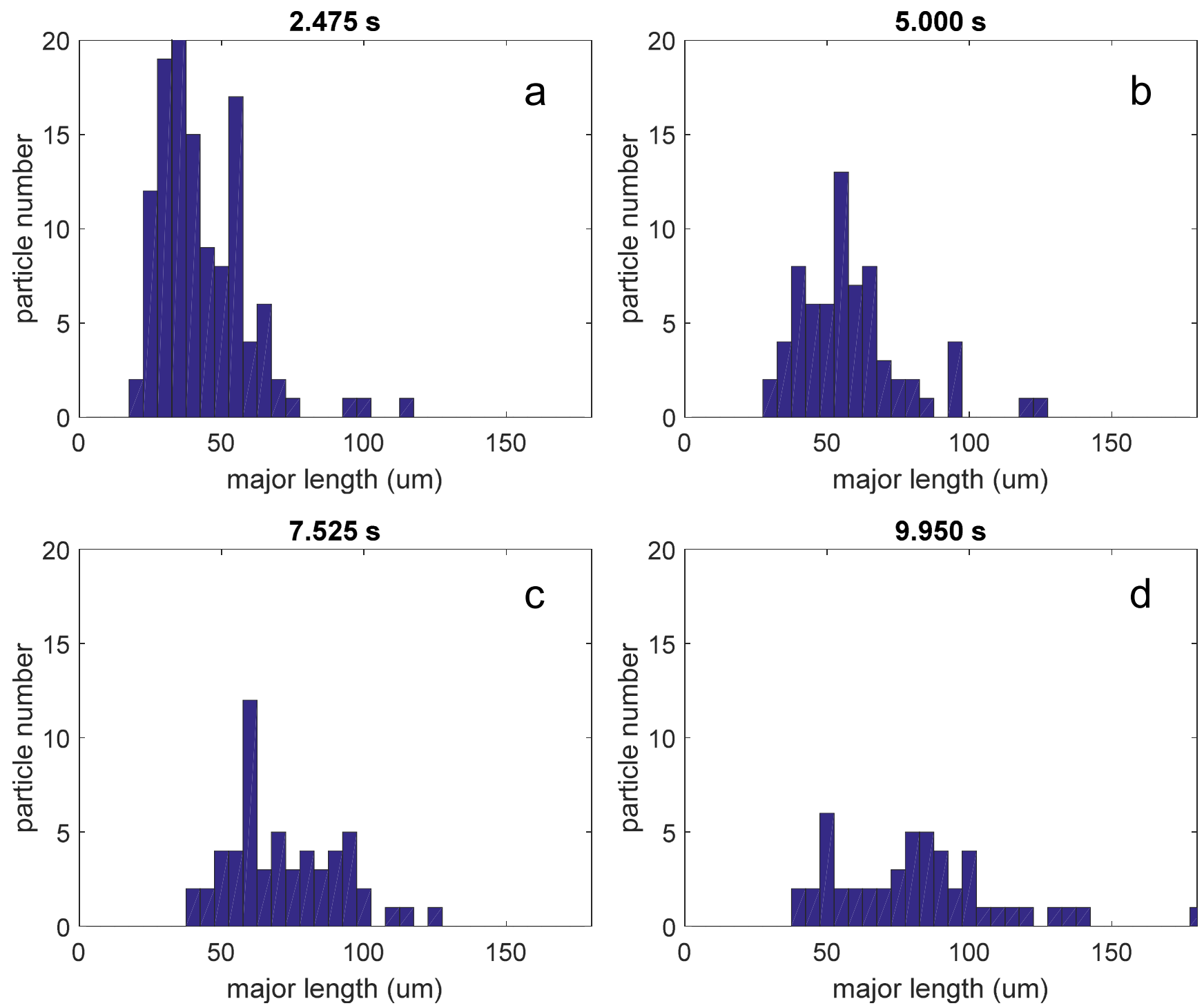

Figure 6. Ice grain major axis length distribution at (a) $2.5 \mathrm{~s}$, (b) $5 \mathrm{~s}$, (c) $7.5 \mathrm{~s}$, and (d) $10 \mathrm{~s}$.

were obtained by analyzing 250 movie frames. A correlation function was used to trace ice grains between two successive frames. Not all the grains can be followed because some grains become out of focus and some grains cannot be distinguished from others. As a result, only ice grains with a unique shape or sharp edges were followed. However, since there is nothing special about this subset, it represents a reasonable sample. Figures 8(a)-(d) show that the mean speed of ice grains becomes slower and the FWHM of the speed distribution becomes narrower with time. This indicates that ice grains become slower as they grow which supports the findings presented in Figure 7(e).

\subsection{Global Behavior}

In order to obtain information on how the ice grains grow throughout the entire plasma volume, a lens with a larger field of view was used to make a movie of the growth process; this movie is shown in Figure 9. The experimental parameters were identical to those used in Figure 3. The ice grains are observed to grow in size as the pressure decreases from 800 mTorr to 400 mTorr, as indicated by Figure 3. It is further observed that dust acoustic waves only occur in the center of the plasma for a short period of time while the ice grains show a flow-like behavior throughout the plasma almost the entire time. It is interesting to note that larger ice grains are observed near the water vapor inlet, which is located on extreme left in the movie images. The observation that dust acoustic waves only occurred in a small central portion of the plasma further indicates that the ice grains likely grow by accretion. Dust acoustic waves have been proposed to be the primary source that provides kinetic energy for ice grains to overcome the Coulomb repulsive energy in laboratory plasmas (Du et al. 2010; Yousefi et al. 2014). However, because the dust acoustic waves do not exist during the entire time that the ice grains grow and are localized to a small central region, it is likely that dust acoustic waves cannot be the reason for the growth here. The appearance of larger ice grains near the water vapor inlet additionally suggests that accretion growth is more important than agglomeration growth.

It is possible that differences between our experiments and those of Du et al. (2010) and Yousefi et al. (2014) account for the different conclusions. For example, we use water vapor spontaneously freezing into ice grains, whereas the other 

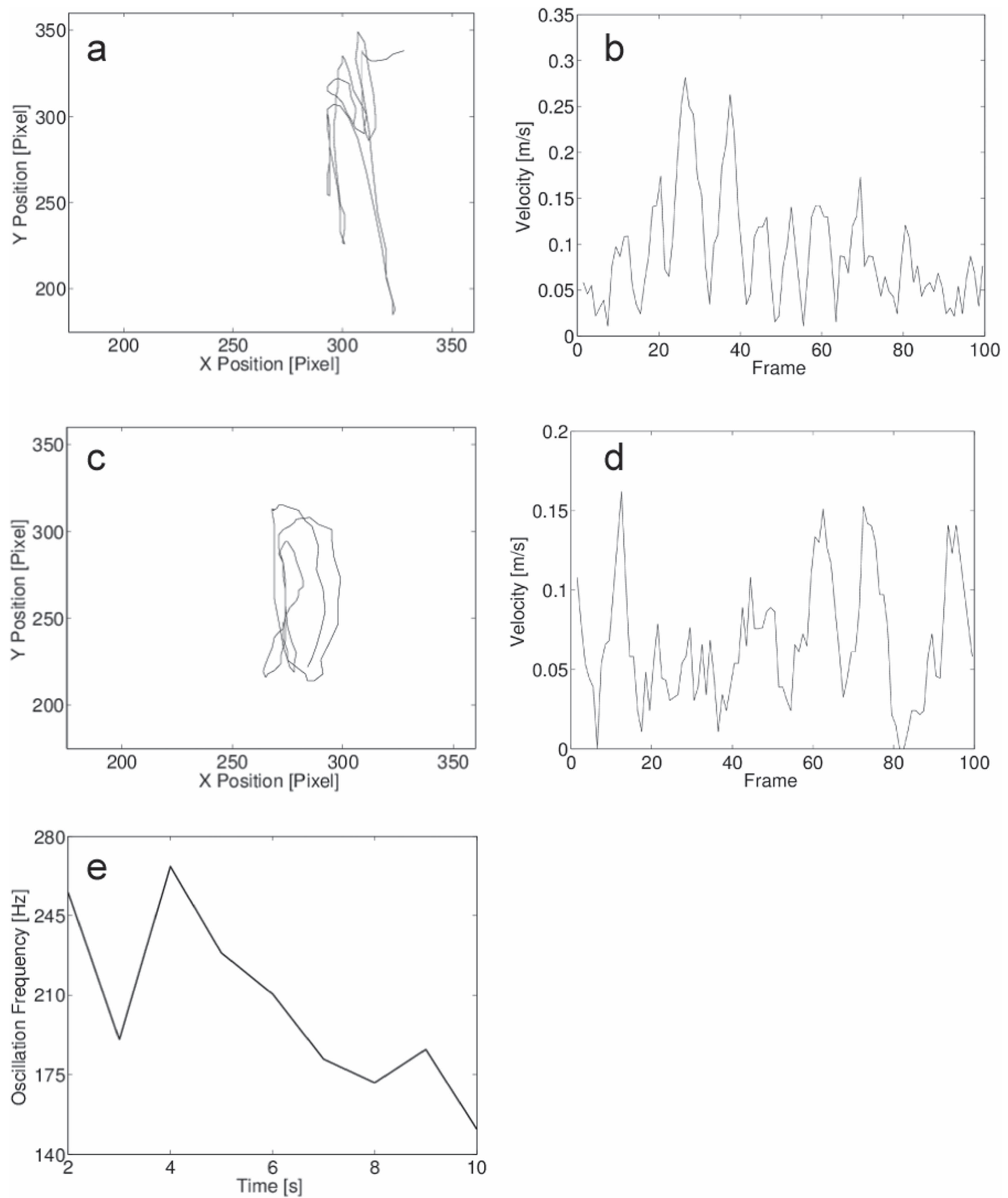

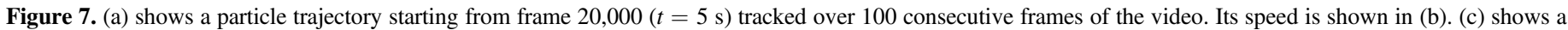

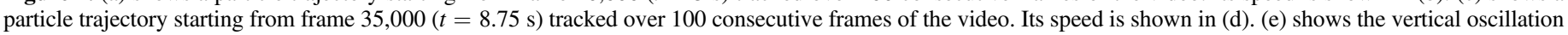
frequency of the ice grains at various times in the video.

experiments start with pre-formed micrometer sized spherical objects that are introduced into the plasma and so cannot undergo accretion. Another difference is that the dust particles were observed to levitate near the plasma-sheath edge in other experiments, whereas the ice grains in our experiment levitate in the bulk plasma region.

Figure 9 also reveals that the ice grains levitate near the top electrode not the bottom electrode. This is different from 

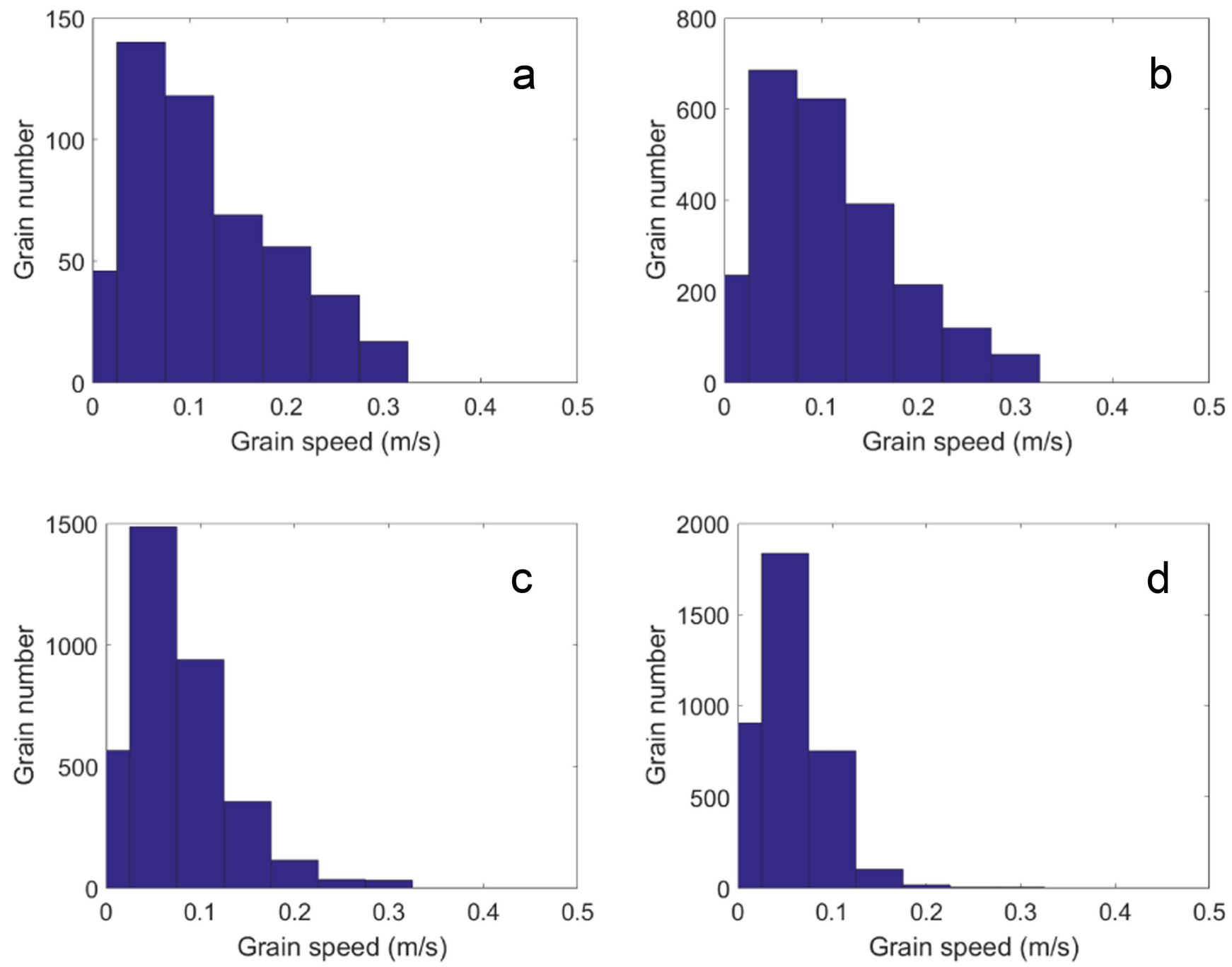

Figure 8. Ice grain speed distribution obtained at (a) 2.5, (b) 5, (c) 7.5, (d) $10 \mathrm{~s}$.

typical laboratory dusty plasma experiments where micron-size dust particles levitate at the plasma-sheath edge near the bottom electrode. This indicates that the downward gravitational force exerted on the ice grains is overwhelmed by the upward thermophoretic force that results from the temperature difference between the top and bottom electrodes; the bottom electrodes are warmer than the top electrode in our experiment.

\section{Discussion}

\subsection{Ice Grain Size and Number Density}

The relationship between ice grain size and ice grain number density provides insight into the dominant growth mechanism. The micron-sized ice grains recorded in the $10 \mathrm{~s}$ video grow in length by a factor of approximately four, while the aspect ratio stays nearly constant. Also, it is presumed that new ice grains are not created in the plasma since we observed that ice grain nucleation ceases at ambient gas pressure lower than 600 mTorr, as stated in Section 2.

If it is supposed that agglomeration causes grain growth, and assuming that this growth is independent of direction, the number density of ice grains should then drop by a factor of $4^{d}$ where $d$ is the fractal dimension of the ice grains. Fractal dimension is used instead of a cubic relation because the ice grains generated are observed to be fractal and the volume of a fractal entity varies as $r^{d}$. The nominal fractal dimension was determined from analysis of $2 \mathrm{D}$ ice grain images in Chai \& Bellan (2015a) to be $d=1.7$. Since the 2D projection of the fractal dimension was found to be independent of the angle from which photographs were taken, the fractal dimension of the $3 \mathrm{D}$ grains can be estimated to be $1.7^{3 / 2}=2.2$. The factor of four increase in ice grain size observed here thus corresponds to a factor of $4^{2.2}=21$ increase in volume with corresponding increase in mass. A mass-conserving 21-fold reduction of the ice grain number density is not observed and this violation of mass conservation contradicts the supposition that agglomeration dominates grain growth. Instead a drop in number density by a factor of only four is observed which implies an increase of the total mass of all the ice grains and so is consistent with growth by accretion and some particle losses.

\subsection{Consideration of the Possibility of Wave-induced Collisions}

The movies show that the ice grains oscillate quite coherently in a wave. It has been proposed by $\mathrm{Du}$ et al. (2010) and Yousefi et al. (2014) that wave-induced collisions can cause two charged grains in a dusty plasma to collide with sufficient kinetic energy to overcome their mutual electrostatic 


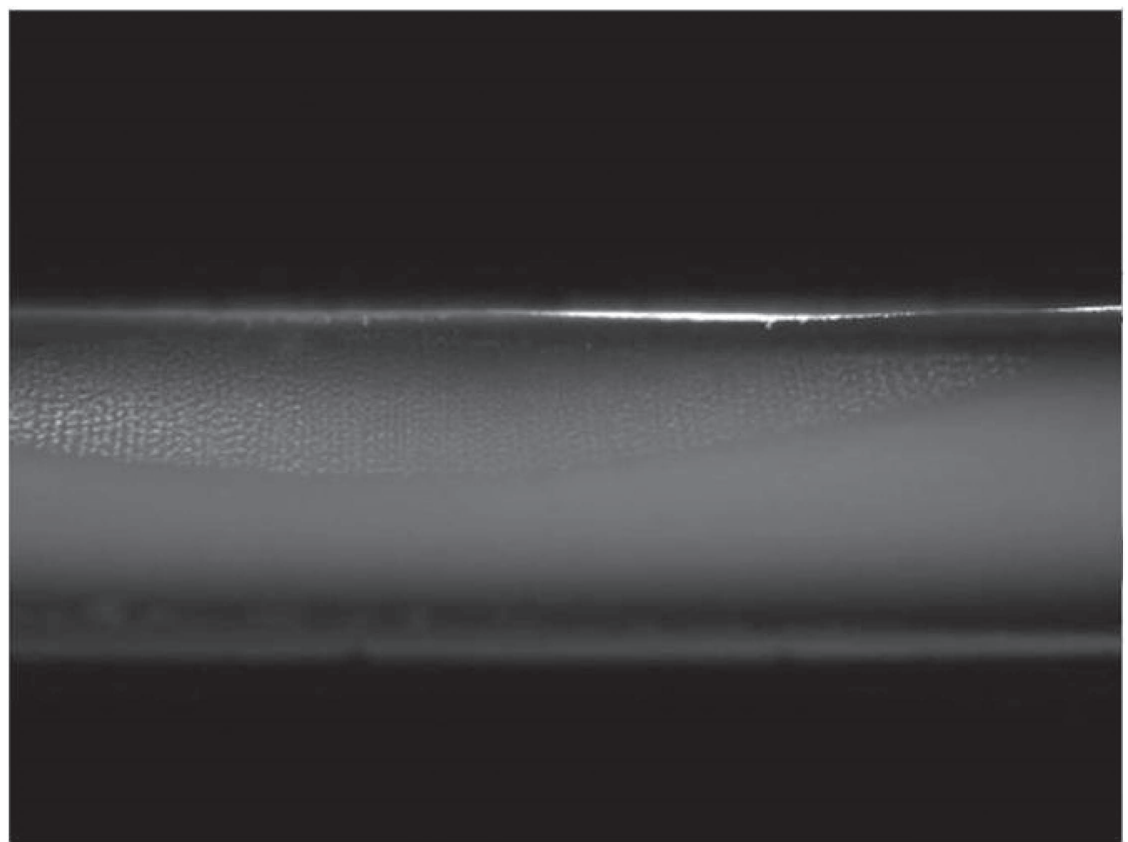

Figure 9. Sample picture from movie taken by Dalsa camera with a wider field of view lens to observe whole plasma dynamics. The movie is slowed by 10 times. (An animation of this figure is available.)

repulsion and so agglomerate. The ice grains are presumed to move in a coherent wave according to the equation of motion

$$
m_{d} \frac{d^{2} y}{d t^{2}}=q_{d} E \cos (k y-\omega t)
$$

and the relevant questions are (i) can two particles starting at different initial positions in this wave collide with each other and (ii) if they collide will the collision be strong enough to overcome mutual electrostatic repulsion. The movies provide the following information: $\omega / 2 \pi=175 \mathrm{~s}^{-1}, 2 \pi / k=2000 \mu \mathrm{m}$, amplitude of ice grain oscillation $\tilde{y}=125 \mu \mathrm{m}$, and nominal ice grain spacing in the compressed region of the wave $\delta=200 \mu \mathrm{m}$. The movies also show that the ice grains have no average velocity, i.e., $\langle d y / d t\rangle=0$ where the angle brackets denote time average.

We now address the above questions using the standard linear analysis of Equation (1). It is first convenient to define the bounce frequency $\omega_{b}=\sqrt{k q_{d} E / m_{d}}$ and the following dimensionless quantities

$$
Y=k y, \tau=\omega_{b} t, \Omega=\omega / \omega_{b}
$$

so Equation (1) becomes

$$
\frac{d^{2} Y}{d \tau^{2}}=\cos (Y-\Omega \tau)
$$

Because $\quad \tilde{Y}=k \tilde{y}=2 \pi \times(125 / 2000)=0.4 \ll \pi / 2 \quad$ we may assume $Y \approx Y_{0}$ in the right-hand side of Equation (3), where $Y_{0}$ is the particle position at $\tau=0$, in which case Equation (3) becomes

$$
\frac{d^{2} Y}{d \tau^{2}}=\cos \left(Y_{0}-\Omega \tau\right)
$$

this is the essential approximation in the standard linear analysis. Integration of Equation (4) gives

$$
\frac{d Y}{d \tau}=V_{0}-\frac{1}{\Omega}\left[\sin \left(Y_{0}-\Omega \tau\right)-\sin Y_{0}\right]
$$

where the first and last terms are constants of integration chosen so that $d Y / d \tau=V_{0}$ at $\tau=0$. In general these constants of integration would cause $\langle d Y / d t\rangle$ to be non-zero, but the observations show that this is not so and so we must choose

$$
V_{0}=-\frac{1}{\Omega} \sin Y_{0}
$$

in which case Equation (5) reduces to

$$
\frac{d Y}{d \tau}=-\frac{1}{\Omega} \sin \left(Y_{0}-\Omega \tau\right) .
$$

Integrating again gives

$$
Y(\tau)=Y_{0}-\frac{1}{\Omega^{2}} \cos \left(Y_{0}-\Omega \tau\right)+\frac{1}{\Omega^{2}} \cos Y_{0}
$$

where the last term is a constant of integration chosen so that $Y=Y_{0}$ at $\tau=0$.

Let us now consider whether two grains initially separated by $\delta$ can collide and for this purpose define the dimensionless separation $\Delta=k \delta$. Consider two particles starting at respective positions $Y_{10}$ and $Y_{20}=Y_{10}+\Delta$ at $\tau=0$ so their respective motions are given by

$$
\begin{aligned}
& Y_{1}(\tau)=Y_{10}-\frac{1}{\Omega^{2}} \cos \left(Y_{10}-\Omega \tau\right)+\frac{1}{\Omega^{2}} \cos Y_{10} \\
& Y_{2}(\tau)=Y_{20}-\frac{1}{\Omega^{2}} \cos \left(Y_{20}-\Omega \tau\right)+\frac{1}{\Omega^{2}} \cos Y_{20} .
\end{aligned}
$$

We now assume that the two particles collide at some collision time $\tau_{c}$, i.e., $Y_{1}\left(\tau_{c}\right)=Y_{2}\left(\tau_{c}\right)$. Subtracting the above two 
equations at this collision time gives

$$
\begin{aligned}
Y_{10} & -Y_{20}-\frac{1}{\Omega^{2}}\left\{\left(\cos \left(Y_{10}-\Omega \tau_{c}\right)-\cos \left(Y_{20}-\Omega \tau_{c}\right)\right)\right. \\
& \left.-\left(\cos Y_{10}-\cos Y_{20}\right)\right\}=0 .
\end{aligned}
$$

Let us define

$$
S=\frac{Y_{10}+Y_{20}}{2}, \quad T=\frac{Y_{10}+Y_{20}}{2}-\Omega \tau_{c}, \quad D=\frac{Y_{10}-Y_{20}}{2}
$$

so $\quad Y_{10}=S+D, \quad Y_{20}=S-D, \quad Y_{10}-\Omega \tau_{c}=T+D$, $Y_{20}-\Omega \tau_{c}=T-D$. Noting that

$$
\cos (S+D)-\cos (S-D)=-2 \sin S \sin D
$$

and similarly for $S \rightarrow T$, Equation (10) can be expressed as

$$
\Delta+\frac{2}{\Omega^{2}}\left\{\sin \left(\bar{Y}_{0}-\Omega \tau_{c}\right)-\sin \left(\bar{Y}_{0}\right)\right\} \sin \left(\frac{\Delta}{2}\right)=0
$$

where $\bar{Y}_{0}=\left(Y_{20}+Y_{10}\right) / 2$. Because $\Delta \ll 1$, Equation (13) simplifies to

$$
\Omega^{2}+\sin \left(\bar{Y}_{0}-\Omega \tau_{c}\right)-\sin \left(\bar{Y}_{0}\right)=0 .
$$

The assumption of small wave amplitude so $Y \approx Y_{0}$ corresponds to having $\Omega^{2} \gg 1$, in which case Equation (14) can never be satisfied. Denoting $\tilde{Y}$ as the oscillatory component of $Y(\tau)$, i.e., the deviation of $Y$ from $Y_{0}$, Equation (8) shows that $|\tilde{Y}|=\Omega^{-2}$. However, the measurements show that $\tilde{Y}=k \tilde{y}=$ $2 \pi \tilde{y} / \lambda=2 \pi \times 125 / 2000=0.4$, so $\Omega^{2}=2.5$ which indicates that Equation (14) cannot be satisfied. We thus conclude that coherent linear wave motion, as described by Equation (4), cannot produce collisions between grains for the observed wave amplitudes. Thus, if the particles collide, the cause must either be random deviations from the linear solution, more complicated waves, or $Y(\tau)$ substantially deviating from $Y_{0}$ in Equation (1), which can happen the wave amplitude were much larger.

\subsubsection{Observational Evidence for Wave-induced Collisions}

If one of the complexities listed above were such that two particles starting at nearly the same position in a wave did collide, their relative velocity would be small because the two particles follow nearly the same trajectory. Figure 10 illustrates this possibility. Two ice grains that started close together were chosen from frame 21,900 of the high-speed video (this frame is at approximately $5.5 \mathrm{~s}$ into the video) and the vertical position of each trajectory for the next 100 frames is plotted in Figure 10.

Figure 10 shows that the $y$ positions of the two particles intersect a few times, indicating that there would be a collision if the particles also had the same $x$ and $z$ positions. Such intersections are at odds with the discussion in Section 4.2, and indicate the existence of a random wave component or that it is incorrect to assume $Y=Y_{0}$ when calculating the wave phase. It is important to note that the trajectories plotted in Figure 10 do not indicate actual collisions because the two ice grains have different $x$ positions and likely different $z$ positions as well. Direct measurements of relative velocities of the very occasional particles that do come close indicate the maximum relative velocity of approaching particles to be $0.20 \mathrm{~m} \mathrm{~s}^{-1}$, and numeric calculations of the wave theory solutions provide a relative velocity of $0.10 \mathrm{~m} \mathrm{~s}^{-1}$. Therefore, $v_{\text {rel }}=0.15 \pm 0.05$ $\mathrm{m} \mathrm{s}^{-1}$ is used for future analysis.

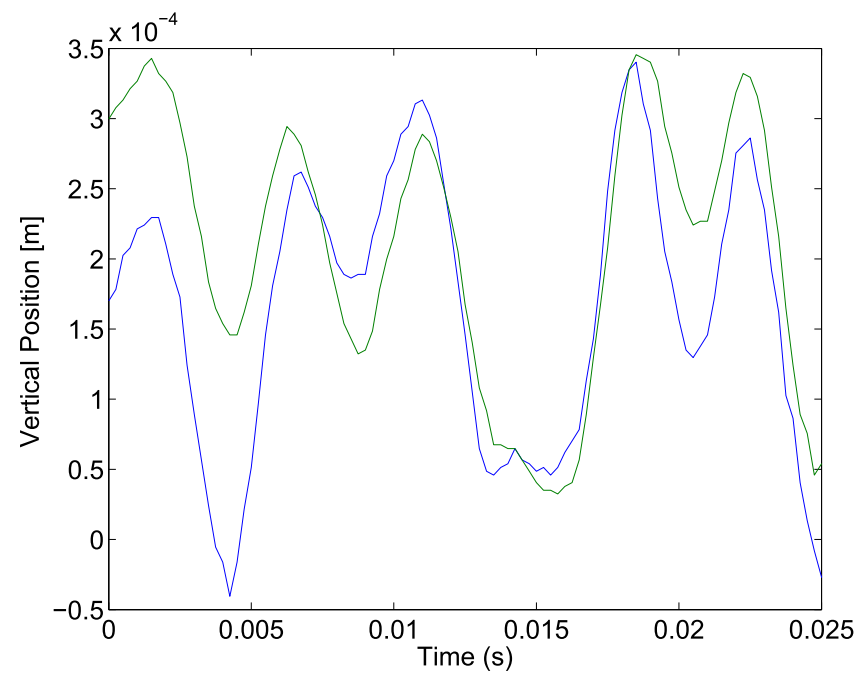

Figure 10. Vertical component of the trajectories of two neighboring ice grains in the plasma starting from frame 21,900 and charting for the next 100 frames. Time $t=0$ at frame 21,900 . Note that when the ice grains occupy the same vertical position (i.e., resulting in a collision in the $1 \mathrm{D}$ model but not in the three dimensions of the actual experiment because the grains are offset horizontally), they have nearly the same vertical velocity (slope), so their relative vertical velocity is very small.

\subsection{Packing Factor}

When two particles approach each other, a comparison between kinetic energy calculated in the center of mass frame and the potential energy associated with Coulomb repulsion provides information on the possibility of collisions. Comparing the initial kinetic energy with the potential energy at closest approach constrains the ice grain packing factor, i.e., the fraction of the ice volume that is solid. The kinetic energy of an ellipsoidal ice grain having major radius $b$, minor radius $a$, and packing factor $p$ is

$$
T=\frac{1}{2} \rho v_{\text {rel }}^{2} \times \frac{4 \pi a^{2} b}{3} \times p
$$

where $v_{\text {rel }}$ is the initial relative velocity between the two ice grains. The potential energy at closest approach $d$ between two dust grains having charge $Z_{d}$ is

$$
U=\frac{Z_{d}^{2} e^{2}}{4 \pi \varepsilon_{0} d}
$$

and at closest approach $T=U$.

Solving $T=U$ for $d$ and noting that no collisions occur if $d>2 b$ gives

$$
Z_{d}>\frac{4 \pi}{e} a b v_{\mathrm{rel}} \sqrt{\frac{p \varepsilon_{0} \rho}{3}} \text { for no collisions. }
$$

The mass density of ice is $\rho=940 \mathrm{~kg} \mathrm{~m}^{-3}$, and $v_{\text {rel }}=(0.15 \pm 0.05) \mathrm{m} \mathrm{s}^{-1}$ will be assumed. We consider nominal grains having $b=20 \mu \mathrm{m}$ and $a=6 \mu \mathrm{m}$. Inserting these values into Equation (17) gives

$$
Z_{d}>(7 \pm 2) \times 10^{4} \sqrt{p} \text { for no collisions. }
$$

An estimate for the packing factor $p$ can be be obtained by calculating $Z_{d}$ from capacitance and floating potential. It is presumed that the dust grain charge lies between the value predicted by a one-dimensional Langmuir probe model (slab model) and a three-dimensional orbital motion limited (OML) 
model. The ice grain floating potential, as determined by onedimensional Langmuir probe theory, is

$$
V_{d}=-T_{e} \ln \left(\frac{m_{i}}{2 \pi m_{e}}\right)^{1 / 2}
$$

The capacitance of an ellipsoidal ice grain is (Chow \& Yovanovich 1982)

$$
C=C_{f} \varepsilon_{0} \sqrt{4 \pi S}
$$

where $S$ is the ellipsoid surface area and $C_{f}$ is a dimensionless factor depending on the elongation $b / a$.

The surface area of a prolate ellipsoid is

$$
S=2 \pi a^{2}\left(1+\frac{\sin ^{-1} \kappa}{\kappa \sqrt{1-\kappa^{2}}}\right)
$$

where

$$
\kappa=\sqrt{1-\frac{a^{2}}{b^{2}}}
$$

so for $a=6 \mu \mathrm{m}$ and $b=20 \mu \mathrm{m}, \quad \kappa=0.954$, giving $S=1.2 \times 10^{-9} \mathrm{~m}^{2}$. Chow \& Yovanovich (1982) gives $C_{f}=1.03$ for $b / a=3.3$. Since $Z_{d} e=C V_{d}$, Equation (19) gives

$$
Z_{d}=C_{f} \varepsilon_{0} \sqrt{4 \pi S} \frac{T_{e}}{e} \ln \left(\frac{m_{i}}{2 \pi m_{e}}\right)^{1 / 2}
$$

so assuming $T_{e}=3 \mathrm{eV}$ for the He plasma, the grain charge calculated using this capacitance method is $Z_{d}=7.4 \times 10^{4}$. We now use $Z_{d}$ calculated from the 1D Langmuir Theory, i.e., Equations (19)-(23), and then after will show the extent to which the results differ if OML theory is used instead to provide a value for $Z_{d}$ in Equation (18).

Combining Equations (17) and (23) and defining the aspect ratio function

$$
F(\kappa)=1-\kappa^{2}+\frac{\sqrt{1-\kappa^{2}}}{\kappa} \sin ^{-1} \kappa
$$

constrains the packing factor to

$$
p<\frac{3 C_{f}^{2} \varepsilon_{0}}{2 \rho} \frac{F(\kappa) T_{e}^{2}}{a^{2} v_{\text {rel }}^{2}}\left(\ln \left(\frac{m_{i}}{2 \pi m_{e}}\right)^{1 / 2}\right)^{2} \text { for no collisions. }
$$

This gives

$$
\begin{aligned}
& p<0.6 \text { for no collisions if } v_{\text {rel }}=0.2 \mathrm{~m} \mathrm{~s}^{-1} \\
& p<2.3 \text { for no collisions if } v_{\text {rel }}=0.1 \mathrm{~m} \mathrm{~s}^{-1}
\end{aligned}
$$

using the values of $\rho, T_{e}, S, a, b, v_{\max }$ given above. For $b / a=1, F(\kappa)=2$, while $F(k) \rightarrow 0$ when $b / a \rightarrow \infty$. For the $a, b$ values used here, $F(\kappa)=0.493$.

If OML theory is used instead of the 1D slab model, then an important parameter is $\alpha=Z_{d} n_{d} / n_{i}$, the fraction of all negative charge in the plasma that is on the dust grains. If $\alpha \rightarrow 0$ is assumed, then Equations (17.13) and (17.27) in Bellan (2006) indicate $Z_{d} \simeq 1.7 \times 10^{4}$ assuming $r_{d}=6 \mu \mathrm{m}$, while if $\alpha=0.2$ is assumed then one obtains $Z_{d} \simeq 1.5 \times 10^{4}$, showing very little sensitivity to the value assumed for $\alpha$, so long as $\alpha$ is small compared to unity. Because Equation (18) shows that $p$ scales as $Z_{d}^{2}$ and because the OML model predicts $Z_{d}$ to be four times smaller than in the 1D slab model, the OML model gives packing factors 16 times smaller, i.e., $p<0.04$ for no collisions if $v_{\text {rel }}=0.2 \mathrm{~m} \mathrm{~s}^{-1}$ and $p<0.14$ for no collisions if $v_{\text {rel }}=0.1 \mathrm{~m} \mathrm{~s}^{-1}$. The lower predicted charge implies less mutual repulsion and so the dust grains would have to be fluffier in order not to collide for the same initial relative velocity.

It is clearly seen from the high-resolution images of ice grains in Figure 2 of Chai \& Bellan (2015a) that, owing to their fractal nature, ice grains formed in our experiment are not completely filled ellipsoid volumes. An estimate based on analysis of the images indicates the packing factor to be $p \approx 0.1$, i.e., an ellipsoid circumscribing the ice grain would have about 10 times the volume of the ice grain. However, the ice grain capacitance would be similar to the ellipsoid because for both the ice grain and the ellipsoid the electric charge from the electrons is concentrated on the extremities because of the mutual repulsion of the electrons. Thus, the observed packing factor is not inconsistent with the estimated constraints on packing factor calculated above.

\subsection{Additional Evidence Supporting Accretion to be the Growth Mechanism}

Two additional experimental observations support the hypothesis that accretion provides the growth mechanism.

The first of these was to vary the water vapor inflow rate and observe any resulting effect on ice grain size; this test is informative because agglomeration should be insensitive to water vapor inflow rate, whereas accretion would be affected. The first step in the experimental sequence was to nucleate ice grains in the He plasma as done for the high-speed movies, i.e., the background $\mathrm{He}$ pressure was started at 1 Torr and then lowered. When the descending background $\mathrm{He}$ pressure crossed $600 \mathrm{mTorr}$, which happened at approximately $t=10 \mathrm{~s}$ as in Figure 2, the water vapor valve was throttled to change the inflow rate. After waiting an additional $10 \mathrm{~s}$ the ice grains were photographed (i.e., at time $20 \mathrm{~s}$ in Figure 2). The experiment was repeated with the valve closed, half open, and fully open. Figure 11(a) shows the initial condition when the background pressure is $600 \mathrm{mTorr}$ (this corresponds to time $10 \mathrm{~s}$ in Figure 2). Figures 11(b)-(d) show the situation for the three different valve settings $10 \mathrm{~s}$ later (i.e., at $20 \mathrm{~s}$ in Figure 2). Figure 11(b) has the valve closed, and the ice grains are very small and spherical; Figure 11(d), which has the valve fully open, has large elongated ice grains, while Figure 11(c) is intermediate. The way the valve was set greatly affected the ice grain size, which is consistent with growth dominated by accretion but not with growth primarily by agglomeration.

The second of these additional observations is given in Chai \& Bellan (2015b). In Section 3.5 of Chai \& Bellan (2015b) smaller and more spherical ice grains are observed when an externally produced $190 \mathrm{G}$ magnetic field is applied to the experiment. This reduction in grain size can be explained by the applied magnetic field causing electrons to undergo cyclotron gyration, in which case the electron flux perpendicular to the magnetic field is attenuated because gyrating electrons cannot move freely across the magnetic field. Because the ions remain essentially unmagnetized, the ion flux is unaffected by the magnetic field. This reduction of the ratio of electron to ion flux on ice grains reduces $Z_{d}$, and because the ice grains are smaller when the magnetic field is applied it can 

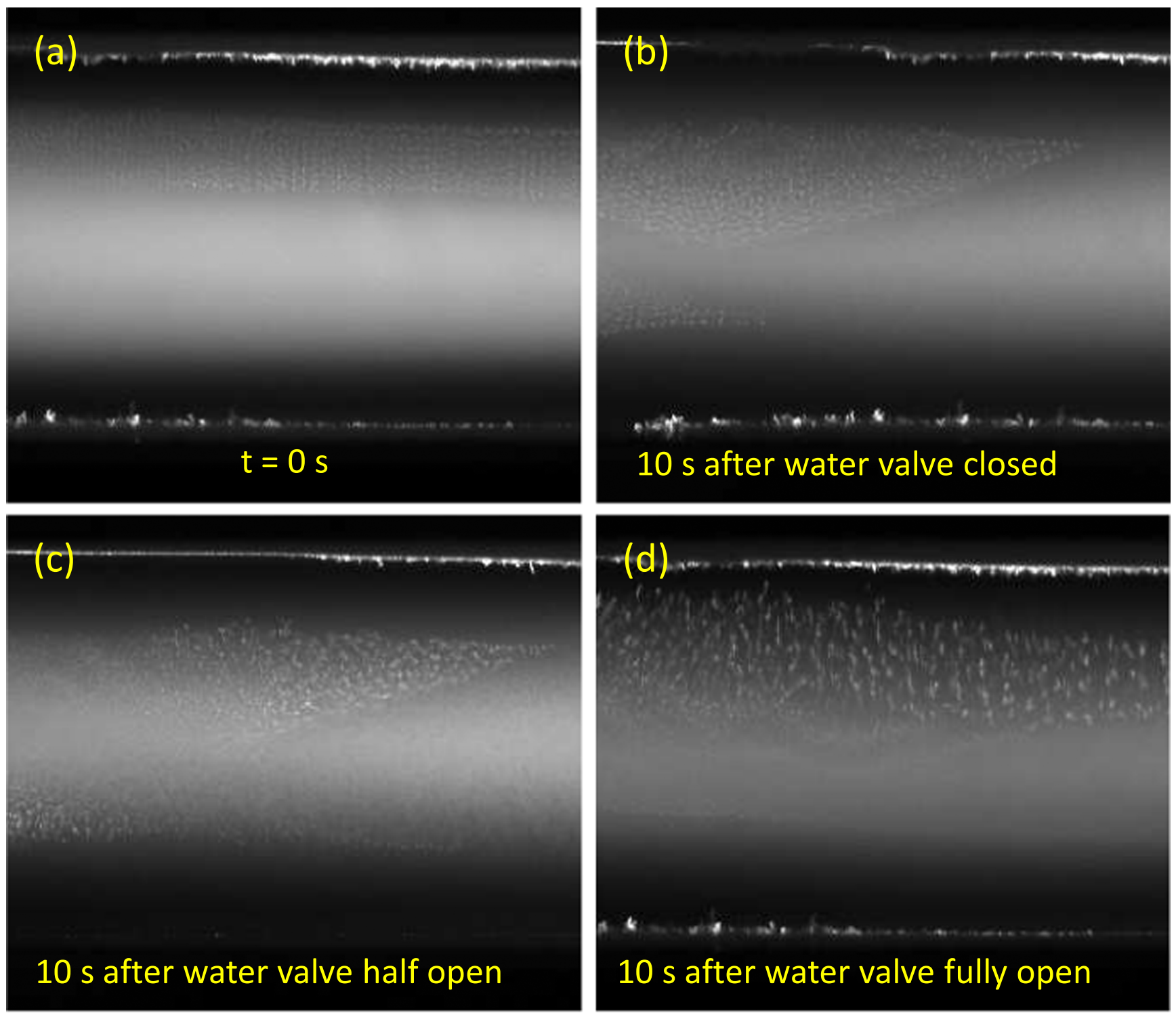

Figure 11. (a) Ice grains captured when the background He pressure was $600 \mathrm{mTorr}$ (at $t=10 \mathrm{~s}$ in Figure 2 ) with water vapor supply. Ice grains imaged $10 \mathrm{~s}$ after (b) water vapor inflow was closed, (c) half opened, and (d) fully opened; the background pressure kept on decreasing as seen in Figure 2 . Larger and more elongated ice grains form with higher water vapor inflow, indicating that accretion is dominant over agglomeration.

be concluded that reduction of $Z_{d}$ results in smaller ice grains. This is the opposite of what would happen if agglomeration were the main growth mechanism because reduction of $Z_{d}$ reduces Coulomb repulsion between grains, which would increase the collision frequency and hence the growth rate by agglomeration.

\subsection{Ice Grain Observation}

The ice grains in this experiment are somewhat smaller than those reported in our previous work (Chai \& Bellan 2015a) for similar conditions. In Chai \& Bellan (2015a) it was stated that ice grains in a low pressure helium plasma attained maximum length of around $300 \mu \mathrm{m}$, whereas here we only see a maximum length of $80 \mu \mathrm{m}$. This discrepancy arises for two reasons. First, in Chai \& Bellan (2015a) the largest ice grains in the plasma were sought out by viewing the entire plasma. Because here the camera field of view is fixed on a small region, it is possible that we are observing a different plasma region where the ice grains are smaller. Second, we are only measuring $10 \mathrm{~s}$ of growth, whereas Chai \& Bellan (2015a) waited one or two minutes until the ice grain growth had saturated. It is highly likely the grains are still growing at the end of the measurement interval in the experiment here.

\subsection{Possible Growth Mechanism for Nanometer Ice Grains}

A growth process scenario consistent with the video observations presented here is that the grains grow by coagulation when they are nanometer-size, but then grow by accreting water vapor when their size exceeds some critical threshold. This two-step process is very similar to the $\mathrm{SiH}_{4}$ and $\mathrm{CH}_{4}$ plasmas in Boufendi \& Bouchoule (1994), Dorier et al. (1995), and Hong et al. (2003). The rationale for the two-step process is that, at nanometer size, because ice grain number density is comparable to the ion number density the water-ice grains cannot be highly charged, so the Coulomb barrier is sufficiently small to overcome. As the ice grains grow by this first-stage agglomeration mechanism, their number density significantly decreases and they become so highly charged that 
the Coulomb barrier becomes unsurpassable. Despite this Coulomb barrier to collisions, it is observed that the ice grains still grow. Because agglomeration cannot be the growth mechanism when there is an insurmountable Coulomb barrier, some other mechanism must take over and the evidence presented here indicates accretion is likely to be this mechanism.

\section{Conclusion}

Analysis of high-speed imaging of water-ice dust grain growth indicates that the charge on the ice grains is important in the micron or greater size regime. This conclusion should be relevant to dust growth in protoplanetary disks from nanometer-size to kilometer-size because the grains must pass through this micron-size regime. If the ice grains are too highly charged to coagulate, as appears to be the case in the lab experiment discussed here, then accretion should be the dominant growth mechanism. The video observations of the ice grain size and number density relationship discussed in Section 4.2 indicate that the accretion mechanism dominates in the larger-than-micron size regime. This conclusion is supported by separate experiments involving variation of the water vapor inflow and applying an external magnetic field to reduce the charge on an ice grain.

In future experiments, we plan to explore the ice grain growth process in the larger-than-micron size regime more quantitatively. We will attempt to develop two measurement techniques to do this. The first measurement would be of the water vapor pressure. While the MKS Capacitance Manometer indicates a pressure increase when the water vapor valve is opened, it is uncertain whether this increase is entirely caused by water vapor. A precise measurement of the water vapor would require a diagnostic that is completely insensitive to the pressure of other constituents; this required specificity to water makes such a measurement extremely difficult. This specificity is essential because there is also some air in the inflow. The air is evident because when the water vapor valve is opened, a typically purple Argon plasma briefly turns pink, the color of a nitrogen plasma for our experimental conditions. The presumption that the water vapor inflow includes air is plausible because nitrogen is the majority constituent of air. The second future measurement would be of the mass of an individual ice grain. This is also a very difficult measurement because of the small size, fragility, and temperature requirements. The comparison of kinetic and potential energy suggests that ice grains have a packing factor much less than unity, but a direct measurement of ice grain mass and volume would confirm this preliminary indication.

This material was based upon work supported by the U.S. Department of Energy Office of Science, Office of Fusion Energy Sciences under Award No. DE-SC0010471.

\section{References}

Andrews, S. M., \& Williams, J. P. 2005, ApJ, 631, 1134

Bellan, P. M. 2006, Fundamentals of Plasma Physics (Cambridge: Cambridge Univ. Press)

Birnstiel, T., Klahr, H., \& Ercolano, B. 2012, A\&A, 539, A148

Blum, J., Wurm, G., Kempf, S., et al. 2000, PhRvL, 85, 2426

Boufendi, L., \& Bouchoule, A. 1994, PSST, 3, 262

Brauer, F., Dullemond, C. P., \& Henning, T. 2008, A\&A, 480, 859

Chai, K. B., \& Bellan, P. M. 2013, GeoRL, 40, 6258

Chai, K. B., \& Bellan, P. M. 2015a, ApJ, 802, 112

Chai, K. B., \& Bellan, P. M. 2015b, JASTP, 127, 83

Chow, Y. L., \& Yovanovich, M. M. 1982, JAP, 53, 8470

Dorier, J. L., Hollenstein, C., \& Howling, A. A. 1995, JVSTA, 13, 918

Du, C. R., Thomas, H. M., Ivlev, A. V., Konopka, U., \& Morfill, G. E. 2010, $\mathrm{PhPl}, 17,113710$

Goertz, C. K. 1989, RvGeo, 27, 271

Greenberg, J. M. 1982, in Submillimeter Wave Astronomy, ed. J. E. Beckman \& J. P. Phillips (New York: Cambridge Univ. Press), 261

Havnes, O., Troim, J., Blix, T., et al. 1996, JGRA, 101, 10839

Hill, T. W., Thomsen, M. F., Tokar, R. L., et al. 2012, JGRA, 117, A05209

Hong, S., Berndt, J., \& Winter, J. 2003, PSST, 12, 46

Jones, G. H., Arridge, C. S., Coates, A. J., et al. 2009, GeoRL, 36, L16204

Kempf, S., Beckmann, U., Moragas-Klostermeyer, G., et al. 2008, Icar, 193,420

Matthews, L. S., Land, V., \& Hyde, T. W. 2012, ApJ, 744, 8

Okuzumi, S. 2009, ApJ, 698, 1122

Okuzumi, S., Tanaka, H., Kobayashi, H., \& Wada, K. 2012, ApJ, 752, 106

Poppe, T., Blum, J., \& Henning, T. 2000, ApJ, 533, 454

Porco, C. C., Helfenstein, P., Thomas, P. C., et al. 2006, Sci, 311, 1393

Ricci, L., Testi, L., Natta, A., et al. 2010, A\&A, 512, A15

Sano, T., Miyama, S. M., Umebayashi, T., \& Nakano, T. 2000, ApJ, 543, 486 Schrapler, R., \& Blum, J. 2011, ApJ, 734, 108

Testi, L., Birnstiel, L., Ricci, L., et al. 2014, in Protostars and Planets VI, ed. H. Beuther et al. (Tucson, AZ: Univ. Arizona Press)

Turco, R. P., Toon, O. B., Whitten, R. C., Keesee, R. G., \& Hollenbach, D. 1982, P\&SS, 30, 1147

Ubach, C., Maddison, S. T., Wright, C. M., et al. 2012, MNRAS, 425, 3137 Yousefi, R., Davis, A. B., Carmona-Reyes, J., Matthews, L. S., \& Hyde, T. W. 2014, PhRvE, 90, 033101 